\title{
Impact of Hyperuricemia on Long-Term Clinical Outcomes of Renal Transplant Recipients: A Systematic Review and Meta-Analysis
}

\author{
Hui Yang ${ }^{1}$, Qing Chen ${ }^{2}$, Aiwen Huang ${ }^{2}$, Xiaojia Yu ${ }^{1}$, Gang Chen ${ }^{1}$, Xiaopeng Hü ${ }^{3}$, Wei Wang ${ }^{3}$, Hang Liu ${ }^{3}$, Xiaodong Zhang, \\ and Lihong Liu ${ }^{1}$
}

${ }^{1}$ Department of Pharmacy, Beijing Chao-Yang Hospital, Capital Medical University, Beijing, China; ${ }^{2}$ Department of Clinical Pharmacy, 900TH Hospital of Joint Logistics Support Force; ${ }^{3}$ Department of Urology, Beijing Chao-Yang Hospital, Capital Medical University, Beijing, China

Corresponding author: Lihong Liu, Pharmacy Department of Beijing Chao-Yang Hospital, 8 Gongren Tiyuchang Nanlu, Chaoyang District, Beijing, China; TEL:(+86) 10 85231077; Fax: (+86) 10 85231077; email: liulihong@bjcyh.com

Received, May 10, 2021; Revised, May 27, 2021; Accepted, May 31, 2021; Published, June 3, 2021

\begin{abstract}
Purpose: To evaluate the effect of hyperuricemia on clinical outcomes of renal transplant recipients (RTRs). Methods: A literature search of PubMed, Cochrane, Embase was conducted up to March 20, 2020. The primary outcome was the estimated glomerular filtration rate (eGFR). The second outcomes were the risk of graft loss, death, cardiovascular event and the level of triglyceride. The following search terms were utilized: ((Hyperuricemic group) OR (Hyperuricaemia) OR (Hyperuric) OR (Urea acid) OR (Uric acid) OR (Acid urate) OR (Urate) OR (Gout)) and ((Transplantation) OR (Transplantations) OR (Transplant) OR (Transplants) OR (Graft)). Results: 28 studies with 18224 patients were eligible for inclusion. There was no significant difference in eGFR $(<12$ months, $\mathrm{p}=0.07)$, the risk of graft loss $(<60$ months, $\mathrm{p}=0.07)$ and death $(<60$ months, $\mathrm{p}=0.19)$ between the hyperuricemic and normouricemic group in the early post-transplantation period. But increased uric acid levels contributed to the long-term decline of eGFR, the risk of graft loss and death increased after transplantation. Hyperuricemia increased the risk of cardiovascular event with no significant difference in the level of triglyceride between the two groups. Conclusions: Increased uric acid levels contributed to the long-term decline of eGFR, increased risk of graft loss and death after transplantation. Although there was no significant effect on triglyceride, hyperuricemia increased the risk of cardiovascular event.
\end{abstract}

\section{INTRODUCTION}

Kidney transplantation is considered the best treatment for patients with end-stage renal disease. However, there are many complications after transplantation, such as hypertension, dyslipidemia, obesity, diabetes, bone metabolism, blood system problems, malignant tumor, electrolyte and acidbase balance disorder and hyperuricemia. It is reported that the incidence of hyperuricemia in renal transplant recipients ranged from $25 \%$ to $84 \%$ (1). The risk factors of hyperuricemia after transplantation are: a decreased estimated glomerular filtration rate (eGFR), diuretic use, cyclosporine therapy, increasing age of the transplant, obesity, metabolic syndrome, as well as the presence of pre-transplant hyperuricemia (2).

In recent years, many studies have been published dealing with the impact of hyperuricemia on the clinical outcomes of renal transplant recipients (RTRs). However, whether hyperuricemia is an independent risk factor, or a marker of progressive graft dysfunction remains a controversial topic (3-4). Huang et al conducted a meta-analysis which included 12 cohort studies in 2012 and found that RTRs with hyperuricemia had a lower estimated glomerular filtration rate (eGFR) and higher serum creatine (SCr) than those with a normal uric acid level. Meta-analysis showed that hyperuricemia might be a risk factor of chronic allograft nephropathy and graft loss (5). However, due to the small sample size of meta-analysis, no clear conclusion could be drawn. In addition, they did not take into account the relationship between the duration of hyperuricemia since transplantation and the clinical outcomes. Many new studies with large samples and comprehensive clinical outcomes have been published in recent years (6-25). Therefore, it is important and necessary to systematically investigate the clinical effect of hyperuricemia on the RTRs in order to produce an evidence-based recommendation for clinical practice.

\section{METHODS}

\section{Search Strategy}

Relevant studies included in this study were identified using PubMed, Cochrane and Embase (from inception up to March 20, 2020). References of relevant articles were also reviewed. The following search terms were utilized: ((Hyperuricemic group) OR (Hyperuricaemia) OR (Hyperuric) OR (Urea acid) OR (Uric acid) OR (Acid urate) OR (Urate) OR (Gout)) and 
((Transplantation) OR (Transplantations) OR (Transplant) OR (Transplants) OR (Graft)). No language restriction was applied to the search. The details of the search strategy were summarized in the Supplementary Table $1 \mathrm{~s}$.

\section{Study selection}

Studies were included according to the following criteria: (1) population-based studies including cohort, case-control, and randomized controlled trial (RCT) studies, and (2) articles that reported the association between hyperuricemia and clinical outcomes in RTRs.

Studies were excluded if the following items were identified: without control group, without available clinical outcomes, and duplicates.

\section{Data extraction}

Two reviewers (QC and HY) independently extracted relevant information for the meta-analysis. The extracted data included the characteristics of each study (author, study design, publication year, country), patient population (numbers of patients, age), length of follow up, definition of hyperuricemic group, time of evaluating eGFR since transplant, adjusted factors and clinical outcomes (glomerular filtration rate, graft loss, overall graft failure, hazard of death, overall survival, cardiovascular event, triglyceride) in each study.

\section{Outcomes}

The primary outcome was eGFR, and the secondary outcomes were the risk of graft loss, death, cardiovascular event, and the level of triglyceride.

\section{Quality assessment}

The methodological quality of the RCTs was evaluated using the criteria developed by the Cochrane risk of bias tool (26): random sequence generation, allocation concealment, blinding of participants and personnel, blinding of outcome assessment, incomplete outcome data, selective reporting, and other bias. The Newcastle-Ottawa Scales (NOS) was used to assess the quality of observational studies (27). Two authors (QC and HY) independently assessed the quality of the studies selected. Discrepancies were resolved by involvement of the third author (XJY).

\section{Statistical analysis}

All statistical analyses were performed using Review Manager for Windows (version 5.3). Mean and standard deviation (SD) were calculated for primary outcomes. Hazard ratio (HR) and 95\% confidence interval (CI) were calculated for secondary outcomes.
Heterogeneity was assessed using the Q statistic and the $\mathrm{I}^{2}$ method. Mantel-Haenszel fixed effects model was used when there was no significant heterogeneity between studies; otherwise, a random effects model was chosen. Publication bias was evaluated using the funnel plot method, of which funnel plot asymmetry was assessed by Egger's linear regression test (28). Sensitivity analysis was performed by exclusion of each study one by one.

\section{RESULTS}

\section{Literature search}

2269 titles and abstracts were yielded through search strategy. And 12 articles were retrieved from a systematic review and meta-analysis by Huang et al. A total of 2191 articles were excluded after the review of abstracts, and 43 articles were excluded after full-text review. Overall, 28 studies with 18224 patients were eligible for inclusion in the metaanalysis (6-25), (29-36). The whole literature search process was summarized in Figure 1.

\section{Study description}

There was no RCT studies reporting the effects of hyperuricemia on clinical outcomes of renal transplant recipients, therefore this meta-analysis was based on the comparison of observational studies. Characteristics of the eligible studies were presented in Table 1. The clinical outcomes of included studies were presented in Table 2.

\section{Quality of included studies}

Risk of bias was assessed using the NOS for all studies. Nine factors were used to assess study quality according to NOS. 5 studies (6-8), (12), (19) missed two indicators, 13 studies (9), (10), (17), (18), (22), (23), (29), (32-35) missed one indicator, and the other 12 studies (11), (13-16), (20), (21), (24), (25), (30), (31), (36) got the full score. All in all, all included observational studies were of high quality (Supplementary Table 2s.).

\section{eGFR}

Twenty studies contained eGFR data for renal transplant recipients (6), (7), (9-11), (13), (15-18), (20), (23), (29-36). Meta-analysis showed that eGFR was significantly lower in the hyperuricemic group than normouricemic group (11104 patients, $\mathrm{MD}=$ 9.21, 95\% CI $-12.42,-6.00, \mathrm{p}<0.00001$, Figure 2). There was no significant difference between the two groups in the first year after transplantation $(p=0.07)$. But, hyperuricemia was associated with significantly lower eGFR after a year post-transplantation (12-36 months, $\quad \mathrm{p}<0.00001 ; \quad 36-60$ months, $\quad \mathrm{p}<0.0001$; 
Table 1. The characteristics of included studies

\begin{tabular}{|c|c|c|c|c|c|c|c|}
\hline $\begin{array}{l}\text { Author, year, } \\
\text { [reference] }\end{array}$ & $\begin{array}{l}\text { Study design, years, } \\
\text { country }\end{array}$ & $\begin{array}{l}\text { Patients } \\
\text { enrolled } \\
\text { (HG/NG) }\end{array}$ & $\begin{array}{l}\text { Median age } \\
\text { (years) }\end{array}$ & $\begin{array}{l}\text { Follow up } \\
\text { (months) }\end{array}$ & $\begin{array}{l}\text { Definition of } \\
\text { hyperuricemia }\end{array}$ & Data analysis & Adjusted factors \\
\hline $\begin{array}{l}\text { Akalin, 2008, } \\
{[36]}\end{array}$ & $\begin{array}{l}\text { Retrospective cohort } \\
\text { study, 2001.1 - } \\
2004.12 \text {, USA }\end{array}$ & $\begin{array}{l}307 \\
(144 / 163)\end{array}$ & $\begin{array}{l}\mathrm{H}: 50.0 \pm 1.0 \\
\mathrm{~N}: 47.7 \pm 1.0\end{array}$ & 51.6 & $\begin{array}{l}\geq 7.0 \text { in men, } \\
\geq 6.5 \mathrm{mg} / \mathrm{dL} \text { in } \\
\text { women }\end{array}$ & multivariate analysis & $\begin{array}{l}\text { age, race, sex, eGFR, having received a cadaveric } \\
\text { transplant nd cyclosporin use }\end{array}$ \\
\hline $\begin{array}{l}\text { Karbowska, } \\
\text { 2009, [35] }\end{array}$ & $\begin{array}{l}\text { NA, } 2003 \text { - 2006, } \\
\text { Poland }\end{array}$ & $78(48 / 30)$ & $\begin{array}{l}\mathrm{H}: 47.8 \\
\mathrm{~N}: 45.3\end{array}$ & $\begin{array}{l}\mathrm{H}: 30.5 \\
\mathrm{~N}: 32.0\end{array}$ & NS & NS & unadjusted \\
\hline $\begin{array}{l}\text { Min, 2009, } \\
{[33]}\end{array}$ & $\begin{array}{l}\text { Cohort study, } 1999.8 \\
\text { - 2006.7, Korea }\end{array}$ & $\begin{array}{l}281 \\
(121 / 160)\end{array}$ & $\begin{array}{l}\mathrm{H}: 41.2 \pm 12.3 \\
\mathrm{~N}: 39.8 \pm 12.2\end{array}$ & 60 & $\geq 8.0 \mathrm{mg} / \mathrm{dl}$ & multivariate analysis & unadjusted \\
\hline $\begin{array}{l}\text { Bandukwala, } \\
\text { 2009, }[34]\end{array}$ & $\begin{array}{l}\text { NA, } 2005.1-2008.4 \text {, } \\
\text { Canada }\end{array}$ & $\begin{array}{l}405 \\
(180 / 225)\end{array}$ & $\begin{array}{l}\mathrm{H}: 50.2 \pm 11 \\
\mathrm{~N}: 50.3 \pm 12\end{array}$ & $\begin{array}{l}\text { H: } 87.6 \pm \\
72 \\
\text { N: } 72 \pm 72\end{array}$ & $\begin{array}{l}>7.1 \mathrm{mg} / \mathrm{dl} \text { in men; } \\
>6.1 \mathrm{mg} / \mathrm{dl} \text { in } \\
\text { women. }\end{array}$ & multivariate analysis & unadjusted \\
\hline $\begin{array}{l}\text { Kim, 2010, } \\
{[32]}\end{array}$ & $\begin{array}{l}\text { Retrospective cohort } \\
\text { study, } 1990 \text { - 2008, } \\
\text { Korea } \\
\end{array}$ & $356(55 / 301)$ & $\begin{array}{l}\mathrm{H}: 36.80 \pm 10.08 \\
\mathrm{~N}: 39.71 \pm 10.28\end{array}$ & $\begin{array}{l}102.63 \pm \\
27.25\end{array}$ & $\begin{array}{l}\geq 7.0 \mathrm{mg} / \mathrm{dL} \text { in men, } \\
\geq 6.0 \mathrm{mg} / \mathrm{dL} \text { in } \\
\text { women }\end{array}$ & multivariate analysis & unadjusted \\
\hline $\begin{array}{l}\text { Haririan, } \\
\text { 2010, [31] }\end{array}$ & $\begin{array}{l}\text { Retrospective cohort } \\
\text { study, 2000.1 - } \\
2001.12, \text { USA }\end{array}$ & $212(45 / 167)$ & $47.9 \pm 13.7$ & $\begin{array}{l}68.3 \pm \\
27.2\end{array}$ & $\begin{array}{l}>6.5 \mathrm{mg} / \mathrm{dl} \text { for } \\
\text { women, } \\
>7.0 \mathrm{mg} / \mathrm{dl} \text { for men }\end{array}$ & NS & age, retransplantation, diabetes and induction \\
\hline Zou, 2010, [29] & $\begin{array}{l}\text { Retrospective cohort } \\
\text { study, 2003.1 - } \\
\text { 2005.12, China }\end{array}$ & $142(58 / 84)$ & $\begin{array}{l}\text { males: } 40.98 \pm \\
11.09 \text {; females: } \\
40.01 \pm 11.62\end{array}$ & $12-36$ & $\begin{array}{l}>420 \mu \mathrm{mol} / \mathrm{L} \text { in } \\
\text { males, } \\
>380 \mu \mathrm{mol} / \mathrm{L} \text { in } \\
\text { females }\end{array}$ & Univariate analysis & unadjusted \\
\hline $\begin{array}{l}\text { Chung, 2010, } \\
{[30]}\end{array}$ & $\begin{array}{l}\text { Retrospective study, } \\
1996.9 \text { - 2004.8, } \\
\text { Korea }\end{array}$ & $\begin{array}{l}350 \\
(148 / 202)\end{array}$ & $38.8 \pm 10.4$ & $\begin{array}{l}103.4 \pm \\
32.8\end{array}$ & $\begin{array}{l}>7.0 \mathrm{mg} / \mathrm{dl} \text { in males, } \\
>6.0 \mathrm{mg} / \mathrm{dl} \text { in females }\end{array}$ & NS & $\begin{array}{l}\text { age, sex, postoperative recovery pattern, the } \\
\text { presence of diabetes or hypertension, BMI, donor } \\
\text { type, retransplantation, HLA mismatch number, } \\
\text { immunosuppressant type, and acute rejection } \\
\text { episodes }\end{array}$ \\
\hline $\begin{array}{l}\text { Kim, 2011, } \\
{[23]}\end{array}$ & $\begin{array}{l}\text { Retrospective cohort } \\
\text { study, } 1990.1 \text { - } \\
\text { 2009.2, Korea }\end{array}$ & $\begin{array}{l}556 \\
(118 / 438)\end{array}$ & $38.74 \pm 10.08$ & 48 & $\begin{array}{l}>6.0 \mathrm{mg} / \mathrm{dl} \text { for } \\
\text { women; } \\
>7.0 \mathrm{mg} / \mathrm{dl} \text { for men }\end{array}$ & Multivariate analysis & unadjusted \\
\hline $\begin{array}{l}\text { Caliskan, } \\
\text { 2011, [24] }\end{array}$ & $\begin{array}{l}\text { Single-center study, } \\
\text { NA, Turkey }\end{array}$ & $141(28 / 113)$ & $37 \pm 11$ & $>6$ & $\begin{array}{l}>6.5 \mathrm{mg} / \mathrm{dl} \text { for } \\
\text { women, } \\
>7.0 \mathrm{mg} / \mathrm{dl} \text { for men }\end{array}$ & NS & $\begin{array}{l}\text { potential confounding factor affecting left } \\
\text { ventricular mass index } \\
\qquad \text { Table } 1 \text { conti }\end{array}$ \\
\hline
\end{tabular}


J Pharm Pharm Sci (www.cspsCanada.org) 24, 292 - 307, 2021

\begin{tabular}{|c|c|c|c|c|c|c|c|}
\hline $\begin{array}{l}\text { Haririan, } \\
\text { 2011, [25] }\end{array}$ & $\begin{array}{l}\text { Retrospective cohort } \\
\text { study, 2004.1 - } \\
\text { 2006.6, USA }\end{array}$ & 488 (NA) & $52.6 \pm 13.1$ & $\begin{array}{l}41.1 \pm \\
17.7\end{array}$ & NS & Univariate analysis & $\begin{array}{l}\text { eGFR, race, donor, peak-PRA, HLA-mismatch, } \\
\text { delayed graft function, acute cellular rejection, } \\
\text { MMF dose, ACEI/ARB }\end{array}$ \\
\hline $\begin{array}{l}\text { Choi, 2013, } \\
\text { [22] }\end{array}$ & $\begin{array}{l}\text { Retrospectively } \\
\text { cohort study, } 1991.4 \text { - } \\
2011.5, \text { Korea }\end{array}$ & $\begin{array}{l}378 \\
(152 / 226)\end{array}$ & $39.37 \pm 10.50$ & $\begin{array}{l}132.36 \pm \\
69.49\end{array}$ & $>7 \mathrm{mg} / \mathrm{dL}$ & $\begin{array}{l}\text { Multivariable } \\
\text { analysis }\end{array}$ & unadjusted \\
\hline $\begin{array}{l}\text { Weng, 2014, } \\
\text { [19] }\end{array}$ & $\begin{array}{l}\text { Prospective cohort } \\
\text { study, } 1999.12 \text { - } \\
\text { 2013.3, China } \\
\end{array}$ & $\begin{array}{l}880 \\
(389 / 491)\end{array}$ & $\begin{array}{l}\mathrm{H}: 50.03 \pm 12.07 \\
\mathrm{~N}: 47.59 \pm 12.57\end{array}$ & $\begin{array}{l}43.3 \pm \\
26.3\end{array}$ & NA & $\begin{array}{l}\text { Time-Varying } \\
\text { Analysis }\end{array}$ & unadjusted \\
\hline $\begin{array}{l}\text { Weng, 2014, } \\
{[20]}\end{array}$ & $\begin{array}{l}\text { Prospective, case- } \\
\text { control study, 2010.9 } \\
-2012.12, \text { China } \\
\end{array}$ & $124(57 / 67)$ & $\begin{array}{l}\mathrm{H}: 50.92 \pm 11.30, \\
\mathrm{~N}: 48.05 \pm 11.52\end{array}$ & 14.27 & NA & Multivariate analysis & age, gender and body mass index. \\
\hline $\begin{array}{l}\text { Dahle, 2014, } \\
\text { [21] }\end{array}$ & $\begin{array}{l}\text { Cohort study, } 2000 \text { - } \\
\text { 2011, Norway }\end{array}$ & $\begin{array}{l}881 \\
(440 / 441)\end{array}$ & 53.0 & 88.8 & NS & $\begin{array}{l}\text { Multivariable } \\
\text { analysis }\end{array}$ & $\begin{array}{l}\text { recipient age, gender, eGFR, diuretics, BMI, } \\
\text { diabetes, impaired glucose tolerance, } \\
\text { cardiovascular disease, number of } \\
\text { antihypertensives, preemptive transplant, first } \\
\text { transplant, donor age, living donor, HLA-DR } \\
\text { mismatches, simultaneous pancreas transplant, } \\
\text { delayed graft function, previous rejection or } \\
\text { cytomegalovirus infection, prednisolone dose, and } \\
\text { immunosuppressive medication. }\end{array}$ \\
\hline $\begin{array}{l}\text { Kim, 2015, } \\
{[14]}\end{array}$ & $\begin{array}{l}\text { Retrospective cohort } \\
\text { study, 2000.1 - } \\
2010.12 \text {, Canada }\end{array}$ & $\begin{array}{l}1170 \\
(247 / 923)\end{array}$ & $49.3 \pm 13.1$ & 120 & $\begin{array}{l}>7.0 \mathrm{mg} / \mathrm{dL} \text { for man, } \\
>6.0 \mathrm{mg} / \mathrm{dL} \text { for } \\
\text { woman }\end{array}$ & $\begin{array}{l}\text { Multivariable Cox } \\
\text { proportional hazards } \\
\text { models }\end{array}$ & $\begin{array}{l}\text { recipient age, sex, race, body mass index, eGFR, } \\
\text { systolic and diastolic blood pressure at baseline, } \\
\text { peak PRA, time on dialysis, donor age, sex, history } \\
\text { of hypertension, body mass index, donor type, } \\
\text { delayed graft function, acute rejection at baseline, } \\
\text { type of CNI at baseline and transplant era. }\end{array}$ \\
\hline $\begin{array}{l}\text { Zhang, 2015, } \\
\text { [15] }\end{array}$ & $\begin{array}{l}\text { Retrospective cohort } \\
\text { study, 2008.1 - } \\
\text { 2011.12, China }\end{array}$ & $\begin{array}{l}573 \\
(155 / 418)\end{array}$ & $41.37 \pm 9.45$ & $\begin{array}{l}41.86 \pm \\
15.49\end{array}$ & $\begin{array}{l}>7.0 \mathrm{mg} / \mathrm{dl} \text { for man, } \\
>6.0 \mathrm{mg} / \mathrm{dl} \text { for } \\
\text { woman }\end{array}$ & $\begin{array}{l}\text { Cox proportional } \\
\text { hazard model, and } \\
\text { multiple regression } \\
\text { equation }\end{array}$ & age, body mass index and male gender \\
\hline $\begin{array}{l}\text { Muela, 2015, } \\
{[16]}\end{array}$ & $\begin{array}{l}\text { Observational study, } \\
1999.7 \text { - 2011.6, } \\
\text { Brazil }\end{array}$ & $199(66 / 133)$ & $52.1 \pm 10.7$ & 19 & NS & $\begin{array}{l}\text { Multivariable } \\
\text { analysis }\end{array}$ & confounding factors, including GFR; \\
\hline $\begin{array}{l}\text { Erkmen Uyar, } \\
2015,[17]\end{array}$ & $\begin{array}{l}\text { Retrospectively } \\
\text { cohort study, } 2008.1 \\
-2010.3, \text { Turkey } \\
\end{array}$ & $100(27 / 73)$ & $38.7 \pm 11$ & $45.9 \pm 9.6$ & $\begin{array}{l}\geq 6.5 \mathrm{mg} / \mathrm{dL} \text { that } \\
\text { persisted for at least } 2 \\
\text { consecutive tests. }\end{array}$ & Multivariate analysis & unadjusted \\
\hline $\begin{array}{l}\text { Fidan, 2015, } \\
{[18]}\end{array}$ & $\begin{array}{l}\text { Retrospectively } \\
\text { cohort study, } 2000.12 \\
-2012.12 \text {, Turkey }\end{array}$ & $81(14 / 67)$ & $16.9 \pm 5.6$ & $42 \pm 5.64$ & $\geq 6 \mathrm{mg} / \mathrm{dL}$ & NS & Table 1 continues... \\
\hline
\end{tabular}


J Pharm Pharm Sci (www.cspsCanada.org) 24, 292 - 307, 2021

\begin{tabular}{|c|c|c|c|c|c|c|c|}
\hline $\begin{array}{l}\text { Han, 2016, } \\
{[12]}\end{array}$ & $\begin{array}{l}\text { Retrospective, multi- } \\
\text { center cohort study, } \\
\text { NA, Korean }\end{array}$ & $\begin{array}{l}2440 \\
(661 / 1779)\end{array}$ & NS & 71.0 & $\begin{array}{l}\geq 7.0 \mathrm{mg} / \mathrm{dL} \text { for } \mathrm{man}, \\
\geq 6.0 \mathrm{mg} / \mathrm{dL} \text { for } \\
\text { woman }\end{array}$ & $\begin{array}{l}\text { Multiple Cox } \\
\text { regression analysis }\end{array}$ & NS \\
\hline Oh, 2016, [13] & $\begin{array}{l}\text { Retrospective cohort } \\
\text { study, } 1991.1 \text { - } \\
2020.12 \text {, Korea }\end{array}$ & $132(70 / 62)$ & $38 \pm 11$ & $199 \pm 5$ & NS & $\begin{array}{l}\text { Multiple linear } \\
\text { regression analysis }\end{array}$ & eGFR \\
\hline $\begin{array}{l}\text { Eyupoglu, } \\
\text { 2017, [9] }\end{array}$ & $\begin{array}{l}\text { Retrospective cohort } \\
\text { study, 2005.12 - } \\
\text { 2016.2, Turkish } \\
\end{array}$ & $141(39 / 102)$ & $37.1 \pm 12.1$ & $\begin{array}{l}83.09 \pm \\
20.30\end{array}$ & $\begin{array}{l}>7.0 \mathrm{mg} / \mathrm{dL} \text { for man, } \\
>6.0 \mathrm{mg} / \mathrm{dL} \text { for } \\
\text { woman }\end{array}$ & Multivariate analyses & NS \\
\hline $\begin{array}{l}\text { Kalil, 2017, } \\
{[10]}\end{array}$ & $\begin{array}{l}\text { Post hoc cohort study, } \\
2002 \text { - 2007, US, } \\
\text { Canada, Brazil }\end{array}$ & $\begin{array}{l}3512 \\
(2398 / 1114)\end{array}$ & $51.7 \pm 69.4$ & 46.8 & $\begin{array}{l}>7 \mathrm{mg} / \mathrm{dL} \text { for man, } \\
>6 \mathrm{mg} / \mathrm{dL} \text { for woman. }\end{array}$ & $\begin{array}{l}\text { Multivariable } \\
\text { analyses }\end{array}$ & NS \\
\hline $\begin{array}{l}\text { Han, 2017, } \\
\text { [11] }\end{array}$ & $\begin{array}{l}\text { Multi-center cohort } \\
\text { study, } 1999.1 \text { - } \\
\text { 2012.8, Korean }\end{array}$ & $\begin{array}{l}1296 \\
(648 / 648)\end{array}$ & $42.1 \pm 11.4$ & $\begin{array}{l}85.9 \pm \\
53.3\end{array}$ & $\begin{array}{l}\geq 7.0 \mathrm{mg} / \mathrm{dL} \text { in males, } \\
\geq 6.0 \mathrm{mg} / \mathrm{dL} \text { in } \\
\text { females }\end{array}$ & Multivariate analyses & Recipient age, gender, eGFR, and donor type. \\
\hline $\begin{array}{l}\text { Ou,2017, } \\
{[8]}\end{array}$ & $\begin{array}{l}\text { Hospital-based cohort } \\
\text { study, } 2010 \text { - 2015, } \\
\text { China }\end{array}$ & $742(\mathrm{NS})$ & NS & NS & NS & NS & NS \\
\hline $\begin{array}{l}\text { Biyik, 2018, } \\
\text { [6] }\end{array}$ & $\begin{array}{l}\text { Retrospective cohort } \\
\text { study, 2015-2017, } \\
\text { Turkey }\end{array}$ & $61(23 / 38)$ & $\begin{array}{l}\mathrm{H}: 47.8 \pm 13.4 \\
\mathrm{~N}: 40.5 \pm 12.9\end{array}$ & $\geq 12$ & $\begin{array}{l}\geq 7 \mathrm{mg} / \mathrm{dL} \text { for man, } \\
\geq 6 \mathrm{mg} / \mathrm{dL} \text { for woman }\end{array}$ & Multivariate analyses & NS \\
\hline $\begin{array}{l}\text { Kim, 2018, } \\
\text { [7] }\end{array}$ & $\begin{array}{l}\text { Retrospective cohort } \\
\text { study, } 1992.1 \text { - } \\
\text { 2014.12, Korea }\end{array}$ & $\begin{array}{l}2198 \\
(676 / 1522)\end{array}$ & $\begin{array}{l}\mathrm{H}: 39.5 \pm 10.9 \\
\mathrm{~N}: 41.3 \pm 11.1\end{array}$ & $160 \pm 64.6$ & $\begin{array}{l}>7.0 \mathrm{mg} / \mathrm{dL} \text { for man, } \\
>6.0 \mathrm{mg} / \mathrm{dL} \text { for } \\
\text { woman }\end{array}$ & Multivariate analyses & NS \\
\hline
\end{tabular}

NS, not stated; NG, normouricemic group; HG, hyperuricemic group; eGFR, estimated glomerular filtration rate; US, United States.

Table 2. The clinical outcomes of included studies

\begin{tabular}{|c|c|c|c|c|c|c|c|c|c|}
\hline \multirow[t]{2}{*}{ Author, year, [Reference] } & \multicolumn{3}{|c|}{ Glomerular filtration rate } & \multirow{2}{*}{$\begin{array}{c}\text { Graft loss } \\
\text { HR }(95 \% \mathrm{CI}) \\
\end{array}$} & \multirow{2}{*}{$\begin{array}{c}\text { Death } \\
\text { HR }(95 \% \mathrm{CI}) \\
\end{array}$} & \multirow{2}{*}{$\begin{array}{c}\begin{array}{c}\text { Cardiovascular } \\
\text { event }\end{array} \\
\text { HR }(95 \% \mathrm{CI})\end{array}$} & \multicolumn{3}{|c|}{ Triglyceride（mg/dL） } \\
\hline & HG & NG & $\mathrm{p}$ & & & & HG & NG & $\mathrm{P}$ \\
\hline Akalin, 2008, [36] & $50.4 \pm 1.3$ & $68.7 \pm 1.6$ & $<0.001$ & NR & NR & $1.70(0.94-3.07)$ & NR & NR & NR \\
\hline Karbowska, 2009, [35] & $68.8 \pm 17.2$ & $76.9 \pm 17.58$ & NR & NR & NR & NR & NR & NR & NR \\
\hline Bandukwala, 2009, [34] & $45.1 \pm 16$ & $58.5 \pm 17$ & $<0.0001$ & NR & NR & $5.62(1.94-16.28)$ & NR & NR & NR \\
\hline Min, 2009, [33] & $73.3 \pm 13.1$ & $71.6 \pm 9.5$ & NR & $2.01(1.09-3.72)$ & NR & NR & NR & NR & NR \\
\hline Kim, 2010, [32] & $73.56 \pm 8.84$ & $78.33 \pm 13.83$ & 0.014 & NR & NR & NR & NR & NR & NR \\
\hline
\end{tabular}


J Pharm Pharm Sci (www.cspsCanada.org) 24, 292 - 307, 2021

\begin{tabular}{|c|c|c|c|c|c|c|c|c|c|}
\hline Haririan, 2010, [31] & $43.2 \pm 15.9$ & $56.1 \pm 19.4$ & NR & $1.92(1.1-3.4)$ & $1.39(0.8-3.0)$ & NR & NR & NR & NR \\
\hline Chung, 2010, [30] & $68.3 \pm 20.4$ & $77.9 \pm 19.2$ & 0.00001 & $2.3(0.9-5.8)$ & $2.37(1.34-4.22)$ & $2.8(1.1-7.1)$ & NR & NR & NR \\
\hline Zou, 2010, [29] & $29.99 \pm 13.05$ & $46.59 \pm 23.21$ & $<0.05$ & NR & NR & NR & NR & NR & NR \\
\hline Haririan, 2011, [25] & NR & NR & NR & $1.15(1.05-1.27)$ & NR & NR & NR & NR & NR \\
\hline Caliskan, 2011, [24] & NR & NR & NR & NR & NR & NR & $150 \pm 57$ & $166 \pm 88$ & 0.368 \\
\hline Kim, 2011, [23] & $56.48 \pm 14.38$ & $65.06 \pm 17.14$ & $<0.001$ & NR & NR & NR & NR & NR & NR \\
\hline Choi, 2013, [22] & NR & NR & NR & NR & $1.86(1.16-2.99)$ & $2.79(0.73-10.71)$ & NR & NR & NR \\
\hline Dahle, 2014, [21] & NR & NR & NR & $1.67(1.18-2.37)$ & $1.57(1.09-2.25)$ & NR & NR & NR & NR \\
\hline Weng, 2014, [20] & $25.48 \pm 14.07$ & $27.60 \pm 14.24$ & 0.408 & NR & $1.68(0.94-3.01)$ & NR & NR & NR & NR \\
\hline Weng, 2014, [19] & NR & NR & NR & NR & $1.37(1.06-1.77)$ & NR & NR & NR & NR \\
\hline Fidan, 2015, [18] & $68.03 \pm 21.84$ & $81.70 \pm 42.23$ & $\leqslant 0.05$ & NR & NR & NR & $126.27 \pm 46.01$ & $136.38 \pm 92.74$ & $>0.05$ \\
\hline Erkmen Uyar, 2015, [17] & $55.4 \pm 21.7$ & $68.6 \pm 23.8$ & 0.014 & NR & NR & NR & $205.3 \pm 98.8$ & $167.6 \pm 72.7$ & 0.042 \\
\hline Muela, 2015, [16] & $40.72 \pm 57$ & $54.85 \pm 117$ & 0.0003 & NR & $0.90(0.59-1.34)$ & $1.6(1.03-2.54)$ & NR & NR & NR \\
\hline Zhang, 2015, [15] & $96.0 \pm 26.6$ & $95.51 \pm 19.13$ & 0.0001 & $1.587(0.733-3.435)$ & NR & NR & $1.96 \pm 0.72$ & $1.88 \pm 0.69$ & 0.032 \\
\hline Kim, 2015, [14] & NR & NR & NR & $0.92(0.54-1.59)$ & $1.05(0.52-2.10)$ & NR & NR & NR & NR \\
\hline Oh, 2016, [13] & $60 \pm 19$ & $71 \pm 24$ & $<0.0001$ & $2.668(1.124-6.332)$ & NR & NR & $147 \pm 77$ & $144 \pm 65$ & 0.0181 \\
\hline Han, 2016, [12] & NR & NR & NR & $1.383(1.01-1.894)$ & $0.891(0.504-1.574)$ & $0.965(0.412-2.262)$ & NR & NR & NR \\
\hline Han, 2017, [11] & $61.2 \pm 18.5$ & $62.8 \pm 16.9$ & 0.103 & $1.65(1.13-2.42)$ & $1.44(0.92-2.26)$ & NR & NR & NR & NR \\
\hline Kalil, 2017, [10] & $45.5 \pm 16.0$ & $55.8 \pm 18.5$ & $<0.001$ & $1.04(0.99-1.10)$ & $1.04(0.99-1.10)$ & $1.02(0.97-1.06)$ & NR & NR & NR \\
\hline Eyupoglu, 2017, [9] & $57.62 \pm 14.16$ & $74.14 \pm 15.7$ & $<0.001$ & $0.52(0.06-4.34)$ & $2.66(0.56-12.67)$ & NR & NR & NR & NR \\
\hline $\mathrm{Ou}, 2017,[8]$ & NR & NR & NR & NR & $2.63(1.20-5.93)$ & NR & NR & NR & NR \\
\hline Kim, 2018, [7] & $58.6 \pm 17.4$ & $67.8 \pm 16.9$ & $<0.001$ & $2.27(1.33-3.78)$ & NR & NR & NR & NR & NR \\
\hline Biyik, 2018, [6] & $53.8 \pm 19.7$ & $70.5 \pm 18.9$ & 0.002 & NR & NR & NR & NR & NR & NR \\
\hline
\end{tabular}

HG, hyperuricemic group; NG, normouricemic group; NR, not report. 
$\geqslant 60$ months, $\mathrm{p}=0.0006$, Figure 2). No obvious asymmetry was found in the funnel plot, which indicated that there is no publication bias in the analysis. The results of sensitivity analysis showed that the result was reliable after the exclusion of individual study one by one.

\section{The risk of graft loss}

A total of 13 studies evaluated the risk of graft loss in RTRs (7), (9-15), (21), (25), (30), (31), (33). The results showed that RTRs in hyperuricemic group had significantly higher risk of graft loss than normouricemia group (13674 patients, $\mathrm{HR}=1.43$, 95\%CI 1.23-1.67, $\mathrm{p}<0.00001 ;$ Figure 3). Stratification by months after transplantation showed that no significant difference was found between two groups during the first 60 months posttransplant $(<60$ months, $\mathrm{HR}=1.13,95 \mathrm{CI} 0.99-1.29, \mathrm{p}=0.07$, Figure 3). But since then, the hyperuricemic group had a significantly increased risk of graft loss than normouricemia group with time (60-84 months, $\mathrm{HR}=1.55,95 \% \mathrm{CI} 1.21-1.98, \mathrm{p}=0.0006 ;>84$ months, $\mathrm{HR}=1.69,95 \% \mathrm{CI} 1.29-2.22, \mathrm{p}=0.0001$; Figure 3). Therefore, the results suggested that hyperuricemia increased the risk of graft loss with time. No obvious asymmetry was found in the funnel plot. Sensitivity analysis showed that the result was reliable after the exclusion of individual study one by one.

\section{The risk of death}

A total of 13 studies evaluated the risk of death in RTRs (8-10), (11-12), (14), (16), (19), (20-22), (30), (31). The results showed that uric acid concentration was associated with the risk of death (12325 patients, $\mathrm{HR}=1.42,95 \%$ CI 1.17-1.72, $\mathrm{p}=0.0004$; Figure 4), but the association was only observed after 60 months post-transplantation in subgroup analysis, and the risk increased with time ( $<60$ months, $\mathrm{HR}=1.15,95 \% \mathrm{CI} 0.93-1.42, \mathrm{p}=0.19 ; 60-84$ months, $\mathrm{HR}=1.49$, 95\%CI $1.05-2.11, \mathrm{p}=0.03$; > 84 months, $\mathrm{HR}=1.74$, 95\%CI 1.32-2.30, $\mathrm{p}=0.0001$; Figure 4). No obvious asymmetry was found in the funnel plot. The results of the sensitivity analysis showed that the result was reliable after the exclusion of individual study one by one.

\section{The risk of cardiovascular event}

A total of 7 studies evaluated cardiovascular event data for RTRs (10), (12), (16), (22), (30), (34), (36). Statistical difference was found in the risk of cardiovascular event between the hyperuricemic group and normouricemic group in RTRs (7591 patients, $\mathrm{HR}=1.70,95 \%$ CI 1.11-2.60, $\mathrm{p}=0.01$; Figure 5). No obvious asymmetry was found in the funnel plot, and sensitivity analysis showed that the result was reliable after the exclusion of individual study one by one.

\section{The level of triglyceride}

Five studies evaluated triglyceride level for RTRs (13), (15), (17), (18), (24). The meta-analysis showed that there was no significant difference in the level of triglyceride between the hyperuricemic group and normouricemic group. (1027 patients, MD $=0.08,95 \%$ CI $-0.05-0.21, \mathrm{p}=0.23$; Figure 6 ). No obvious asymmetry was found in the funnel plot, which indicated that there is no publication bias in the analysis. The results of sensitivity analysis showed that the result was reliable.

\section{DISCUSSION}

Hyperuricemia is a common comorbid condition experienced in RTRs (37). It is unclear, however, whether hyperuricemia plays a casual role in the development of graft dysfunction. To the best of our knowledge, this was the most comprehensive systematic review and meta-analysis to evaluate the association between hyperuricemic status and RTRs' long-term outcomes. Besides, we took into account the relationship between the duration of hyperuricemia since transplantation and the clinical outcomes. Our study found that there was no significant difference in eGFR, the risk of graft loss and death at the early time post-transplantation between the hyperuricemic and normouricemic group. However, increased uric acid levels contributed to the long-term decline of eGFR, the risk of graft loss and death increase after transplantation. Hyperuricemia increased the risk of cardiovascular event with no significant difference in the level of triglyceride between the two groups.

Our study showed that hyperuricemia was associated with lower eGFR compared with normal serum UA levels. Previous studies reported that uric acid induced graft dysfunction and chronic allograft nephropathy and accelerated the progress of chronic kidney disease (38-39). The proposed mechanisms included that uric acid was a mediator of endothelial dysfunction, inflammation, and vascular disease (35), (40-41). Our results were similar with previous study, and our study found that this impact was only observed at 12-months following transplantion. Gerhardt et al found that patients with hyperuricemia demonstrated a 5-year graft survival rate of $68.8 \%$, compared with $83.3 \%$ in patients with normouricemia (42). Our studies also found that hyperuricemia post-kidney transplantation reduced graft survival. Several mechanisms for the effect of 


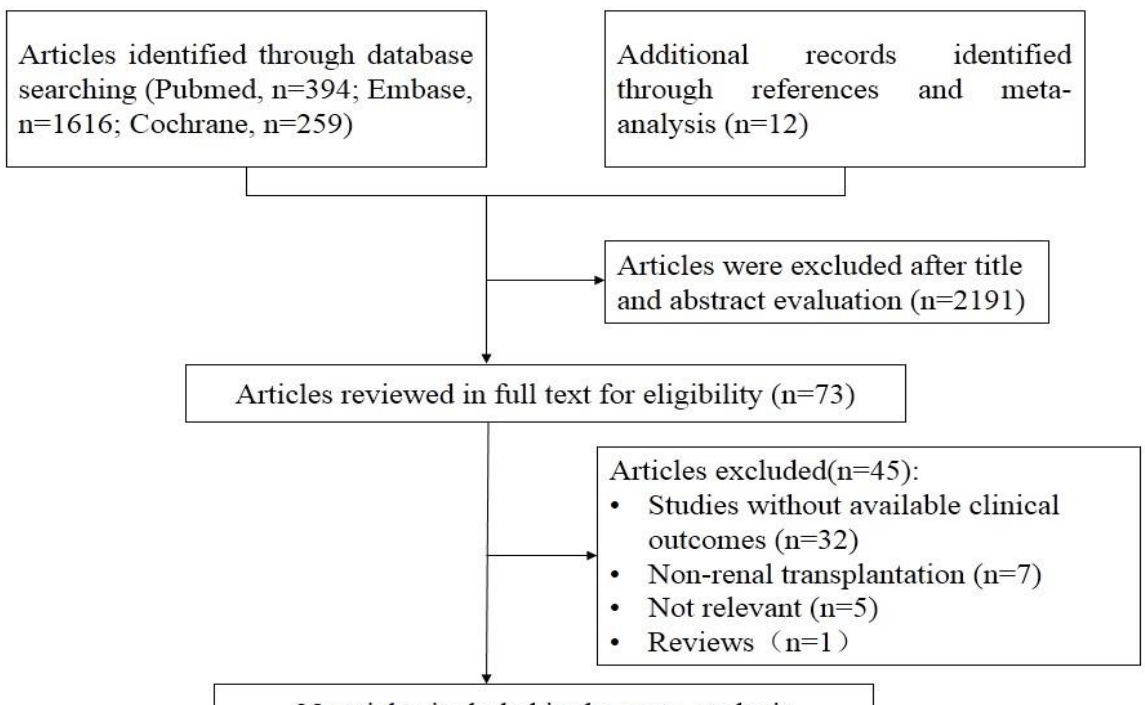

28 articles included in the meta-analysis

Figure 1. Flow chart depicting the selection process of studies included in the meta-analysis.

\begin{tabular}{|c|c|c|c|c|c|c|c|c|c|}
\hline \multirow[b]{2}{*}{ Studv or Subgroup } & \multicolumn{3}{|c|}{ hyperuricemic group } & \multicolumn{3}{|c|}{ normouricemic group } & \multicolumn{3}{|c|}{ Mean Difference } \\
\hline & Mean & SD & Total & Mean & SD & Total & Weight & IV. Random, $95 \% \mathrm{Cl}$ & \\
\hline \multicolumn{10}{|l|}{$7.1 .1<12$ months } \\
\hline Fidan 2015 & 90.86 & 37.32 & 14 & 92.83 & 31.01 & 67 & $1.5 \%$ & $-1.97[-22.88,18.94]$ & 20 \\
\hline Muela 2015 & 40.72 & 57 & 66 & 54.85 & 117 & 133 & $1.3 \%$ & $-14.13[-38.31,10.05]$ & 20 \\
\hline Zhang 2015 & 92.9 & 21.5 & 155 & 95.51 & 19.13 & 418 & $4.2 \%$ & $-2.61[-6.46,1.24]$ & 20 \\
\hline Han 2017 & 61.2 & 18.5 & 648 & 62.8 & 16.9 & 648 & $4.4 \%$ & $-1.60[-3.53,0.33]$ & 201 \\
\hline Kim 2018 & 56.2 & 20.9 & 676 & 65.6 & 19.7 & 1522 & $4.4 \%$ & $-9.40[-11.26,-7.54]$ & \\
\hline Subtotal $(95 \% \mathrm{Cl})$ & & & 1559 & & & 2788 & $15.9 \%$ & $-4.87[-10.10,0.36]$ & \\
\hline \multicolumn{10}{|c|}{$\begin{array}{l}\text { Heterogeneity: Tau }=21.74 ; C^{2} i^{2}=35.27, d f=4(P<0.00001) ; i^{2}=89 \% \\
\text { Test for overall effect: } Z=1.82(P=0.07)\end{array}$} \\
\hline \multicolumn{10}{|l|}{ 7.1.2 12-36 months } \\
\hline Weng 2014 (2) & 25.48 & 14.07 & 57 & 27.6 & 14.24 & 67 & $4.1 \%$ & $-2.12[-7.12,2.88]$ & \\
\hline Karbowska 2009 & 68.8 & 17.2 & 48 & 76.9 & 17.58 & 30 & $3.5 \%$ & $-8.10[-16.05,-0.15]$ & 20 \\
\hline Zou 2010 & 37.38 & 16.6 & 58 & 52.63 & 23.72 & 84 & $3.8 \%$ & $-15.25[-21.88,-8.62]$ & \\
\hline Kim 2011 & 56.48 & 14.38 & 118 & 65.06 & 17.14 & 438 & $4.3 \%$ & $-8.58[-11.63,-5.53]$ & \\
\hline Zhang 2015 & 94.9 & 25.4 & 155 & 95.51 & 19.13 & 418 & $4.2 \%$ & $-0.61[-5.01,3.79]$ & 201 \\
\hline Fidan 2015 & 73.85 & 23.63 & 14 & 92.2 & 30.97 & 67 & $2.4 \%$ & $-18.35[-32.78,-3.92]$ & 20 \\
\hline Erkmen Uyar & 61.4 & 22.5 & 27 & 73.4 & 24.5 & 73 & $3.1 \%$ & $-12.00[-22.18,-1.82]$ & \\
\hline Kim 2 & 58.6 & 17.4 & 676 & 67.8 & 16.9 & 1522 & $4.5 \%$ & $-9.20[-10.76,-7.64]$ & 20 \\
\hline Biyik? & 53.8 & 19.7 & 23 & 70.5 & 18.9 & 38 & $3.1 \%$ & $-16.70[-26$ & \\
\hline Subtotal $(95 \% \mathrm{Cl})$ & & & 1176 & & & 2737 & $32.9 \%$ & & \\
\hline \multirow{2}{*}{\multicolumn{10}{|c|}{$\begin{array}{l}\text { Heterogeneity: Tau }=13.23 ; C h i^{2}=28.17, d f=8(P=0.0004) ; I^{2}=72 \% \\
\text { Test for overall effect: } Z=5.25(P<0.00001)\end{array}$}} \\
\hline & & & & & & & & & \\
\hline \multicolumn{10}{|l|}{ 7.1.3 36-60 months } \\
\hline Akalin 2008 & 50.4 & 1.3 & 144 & 68.7 & 1.6 & 163 & $4.5 \%$ & $-17.98]$ & 20 \\
\hline Zou & 29.99 & 13.05 & 58 & 46.59 & 23.21 & 84 & $3.9 \%$ & -16.6 & 2010 \\
\hline Jyar 2015 & 55.4 & 21.7 & 27 & 68.6 & 23.8 & 73 & $3.2 \%$ & $-13.20[-23.04,-3.36]$ & 20 \\
\hline Fidan & 68.03 & 21.84 & 14 & 81.7 & 42.23 & 67 & $2.2 \%$ & $-13.67[-28.94,1.60]$ & 20 \\
\hline Zhan & 96 & 26.6 & 155 & 95.51 & 19.13 & 418 & $4.1 \%$ & $0.49[-4$ & \\
\hline Kalil 2017 & 45.5 & 16 & 2398 & 55.8 & 18.5 & 1114 & $4.5 \%$ & $-10.30[-11.56,-9.04]$ & 20 \\
\hline Subtotal $(95 \% \mathrm{Cl})$ & & & 2796 & & & 1919 & $22.4 \%$ & $-11.73[-17.40,-6.06]$ & \\
\hline \multirow{2}{*}{\multicolumn{10}{|c|}{$\begin{array}{l}\text { Heterogeneity: } \text { Tau }^{2}=39.07 ; \mathrm{Chi}^{2}=207.41, \mathrm{df}=5(\mathrm{P}<0.00001) ; \mathrm{I}^{2}=98 \% \\
\text { Test for overall effect: } Z=4.06(P<0.0001)\end{array}$}} \\
\hline & & & & & & & & & \\
\hline \multicolumn{10}{|l|}{ 7.1.4 $\geqslant 60$ months } \\
\hline $\operatorname{Min} 2009$ & 73.3 & 13.1 & 121 & 71.6 & 9.5 & 160 & $4.4 \%$ & 4.46] & \\
\hline Band & 45.1 & 16 & 180 & 58.5 & 17 & 225 & $4.3 \%$ & $-13.40[-1$ & \\
\hline Chung 2010 & 68.3 & 20.4 & 148 & 77.9 & 19.2 & 202 & $4.2 \%$ & $-9.60[-13.82,-5.38]$ & \\
\hline Kim 2010 & 73.56 & 8.84 & 55 & 78.33 & 13.83 & 301 & $4.4 \%$ & $-4.77[-7.58,-1.96]$ & 20 \\
\hline Harivian 20 & 43.2 & 15.9 & 45 & 56.1 & 19.4 & 167 & $4.0 \%$ & $-12.90[-18.40,-7.40]$ & \\
\hline On 2016 & 60 & 19 & 70 & 71 & 24 & 62 & $3.6 \%$ & $-11.00[-18.45,-3.55]$ & 20 \\
\hline Eyupo & 57.62 & 14.16 & 39 & 74.14 & 15.7 & 102 & $4.0 \%$ & $-16.52[-21.91,-11.13]$ & \\
\hline Subtotal $(95 \% \mathrm{Cl}$ & & & 658 & & & 1219 & $28.8 \%$ & $-9.27[-14.53,-4.01]$ & \\
\hline \multirow{2}{*}{\multicolumn{10}{|c|}{$\begin{array}{l}\text { Heterogeneity: } \text { Tau }^{2}=44.88 ; \mathrm{Chi}^{2}=74.68, \mathrm{df}=6(\mathrm{P}<0.00001) ; \mathrm{I}^{2}=92 \% \\
\text { Test for overall effect: } Z=3.45(\mathrm{P}=0.0006)\end{array}$}} \\
\hline & & & & & & & & & \\
\hline Total $(95 \% \mathrm{Cl})$ & & & 6189 & & & 8663 & $100.0 \%$ & $-9.21[-12.42,-6.00]$ & \\
\hline \multirow{2}{*}{\multicolumn{10}{|c|}{$\begin{array}{l}\text { Heterogeneity: } \text { Tau }^{2}=59.61 ; C h i^{2}=1042.26, d f=26(P<0.00001) ; i^{2}=98 \% \\
\text { Test for overall effect: } Z=5.62(P<0.00001)\end{array}$}} \\
\hline & & & & & & & & & \\
\hline Test for subaro & 0.01 & & & & & & & & \\
\hline
\end{tabular}

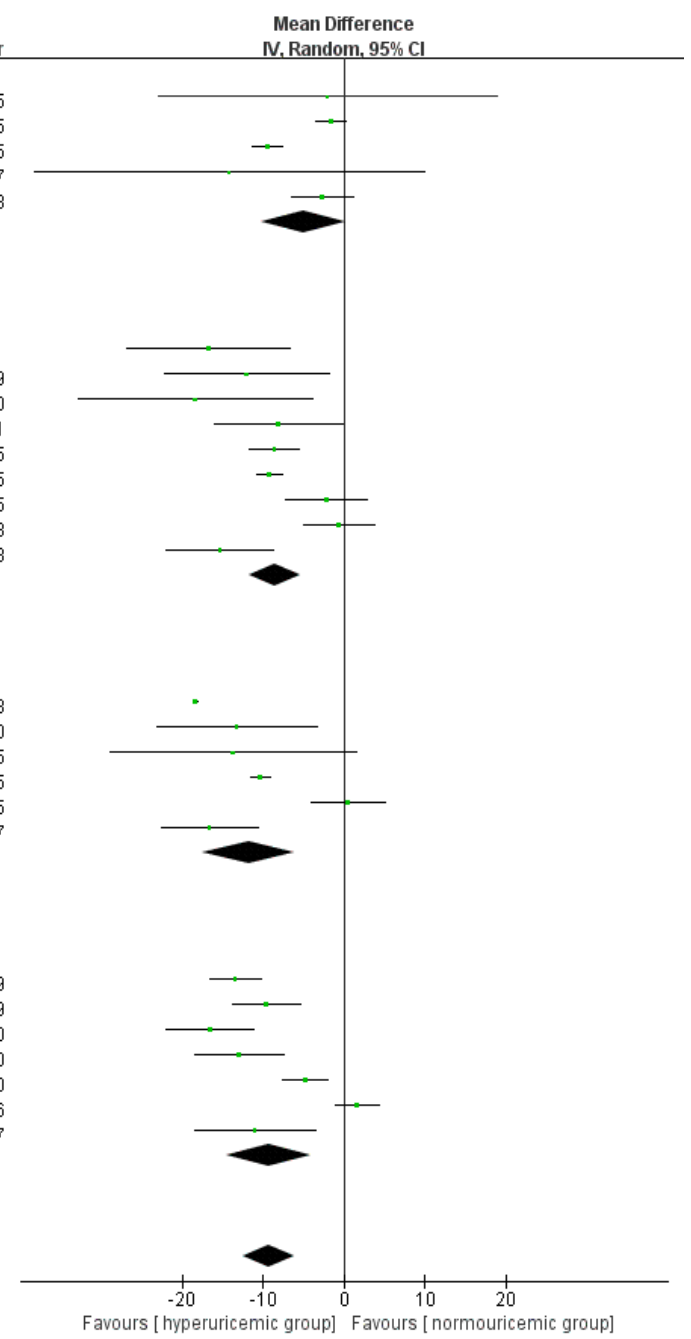

Figure 2. Forest plot for hyperuricemia and eGFR 


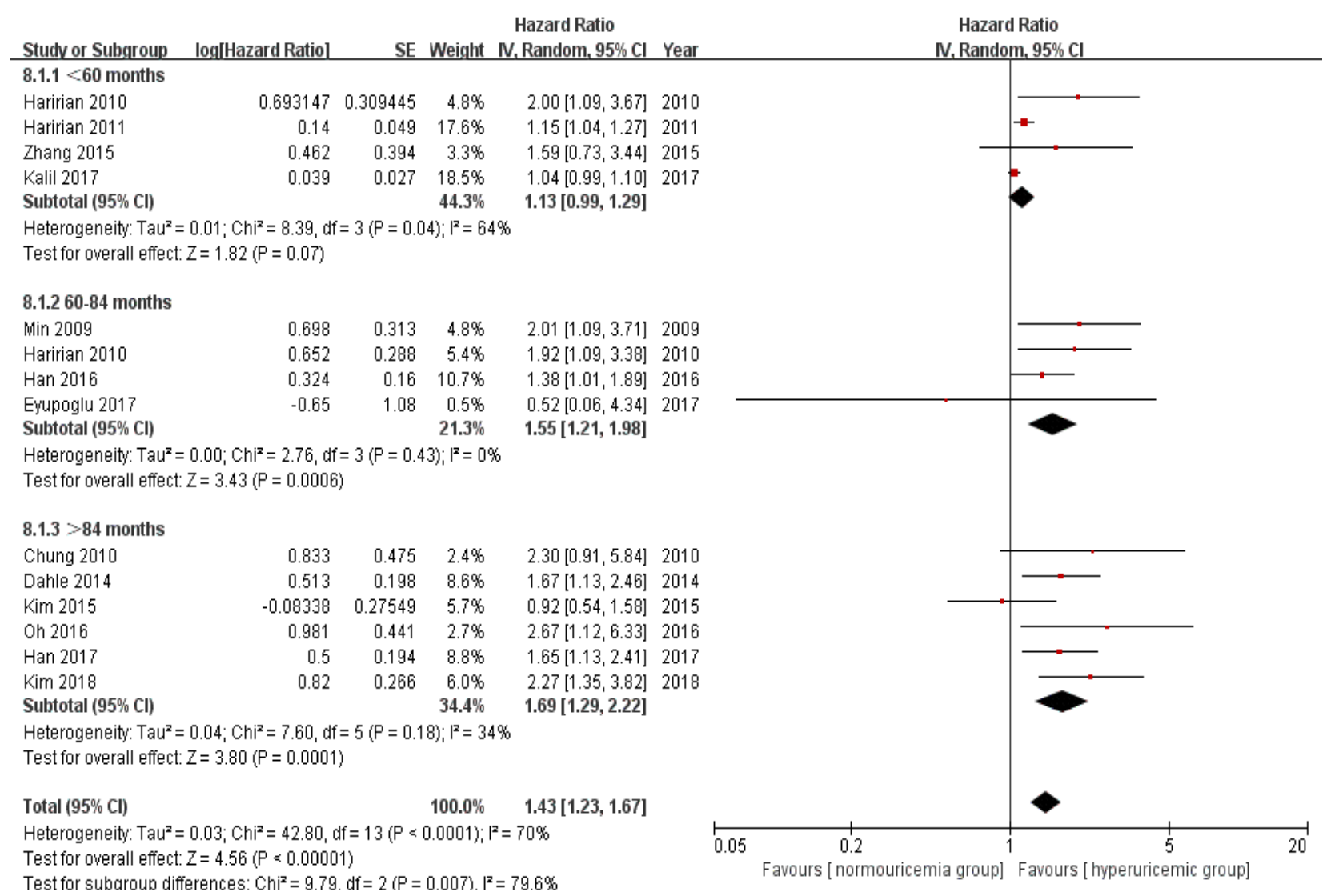

Figure 3. Forest plot for hyperuricemia and the risk of graft loss

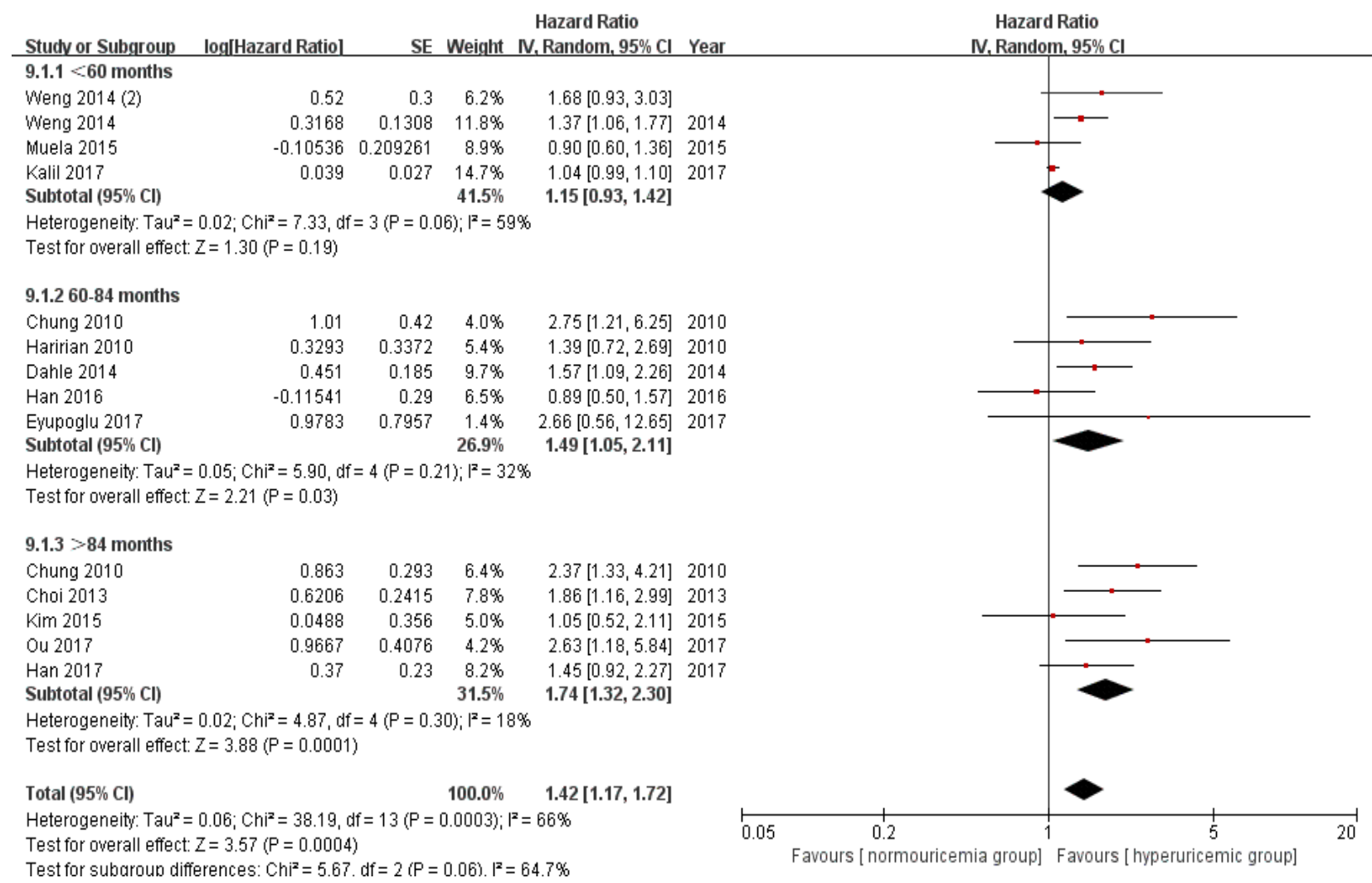

Figure 4. Forest plot for hyperuricemia and the risk of death 


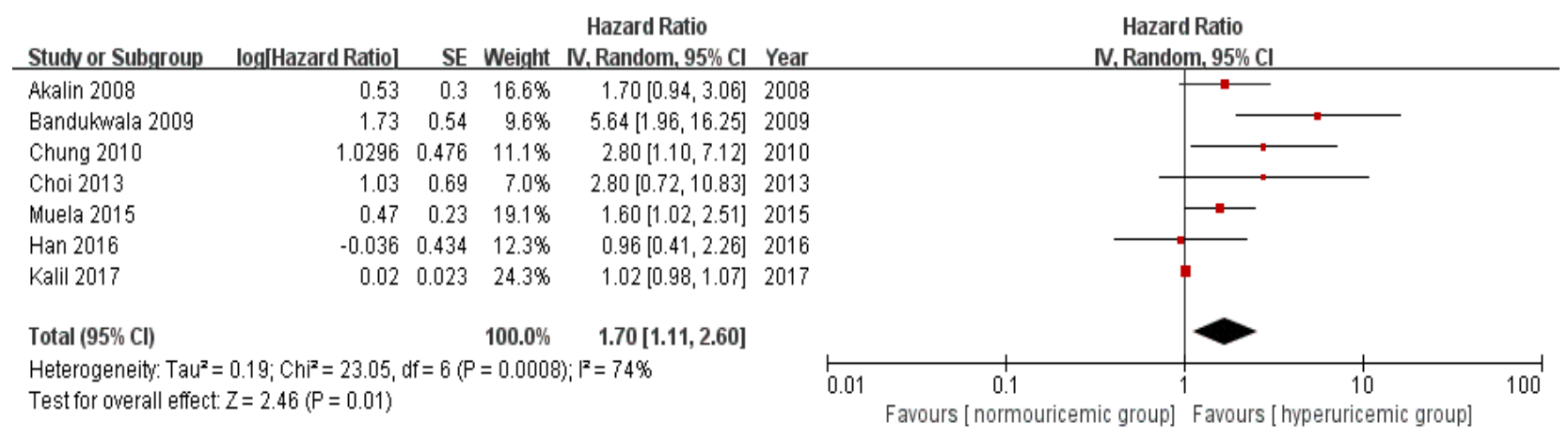

Figure 5. Forest plot for hyperuricemia and the risk of cardiovascular event

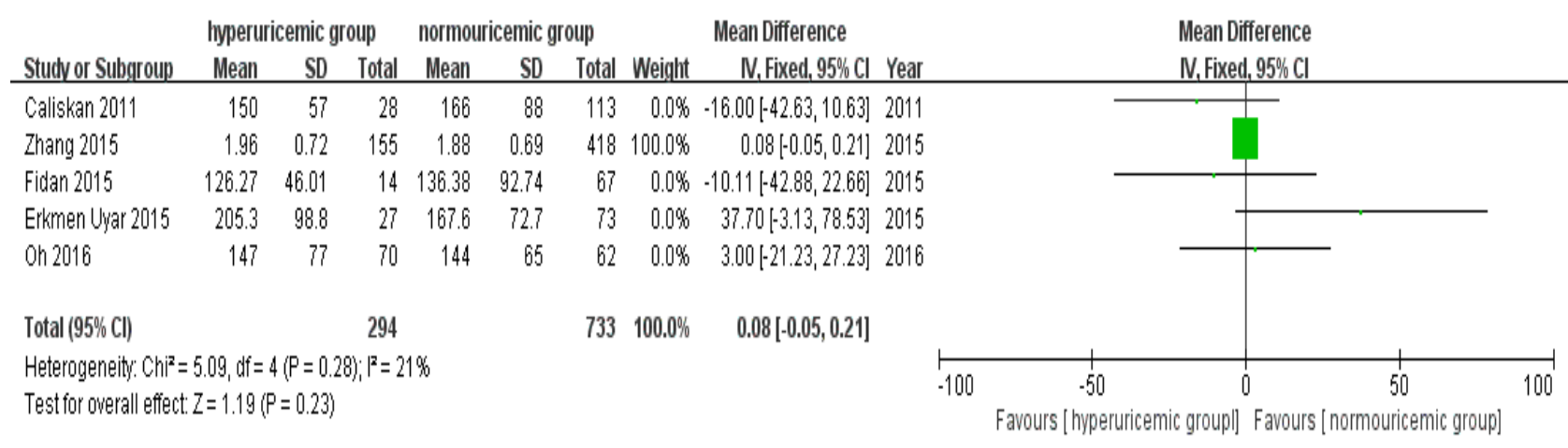

Figure 6. Forest plot for hyperuricemia and the level of triglyceride

UA on graft loss have been proposed. Hyperuricemia induced glomerular hypertrophy (43), renal arteriolopathy (44), endothelial dysfunction (45) and arterial stiffness (46-47). These factors resulted in chronic allograft nephropathy, which were the major causes of late graft loss (48-49). And our study found that the association was only observed at 60 months after operation.

National Health and Nutrition Examination Survey (NHANES) has demonstrated an association between hyperuricemia/gout and cardiovascular disease (50). The uric acid (UA) levels are associated with coronary artery calcium (51). Viazzi et al demonstrated that each standard deviation increase in serum uric acid entailed a $75 \%$ higher risk of having cardiac hypertrophy and a 2-times greater risk of having carotid abnormalities (52). There are many proposed mechanisms for the influence of hyperuricemia on the cardiovascular risks. Uric acid causes cardiovascular disorders by

stimulating the vascular renin-angiotensin systems, serving as a bridging mechanism mediating (enabling) or potentiating the deleterious effects of cardiovascular risk factors on vascular tissue and myocardium (53-54). The impact of uric acid concentrations on cardiovascular event is limited. This was the first meta-analysis to demonstrate this association.

Our study suggested that hyperuricemia increased the risk of poor outcomes of RTRs. Therefore, the treatment of hyperuricemia is essential. But consensus on whether to treat asymptomatic hyperuricemia in CKD has not been established, treatment of asymptomatic hyperuricemia has not been generally recommended in the general population or KTRs, and it is only advocated in those with recurrent symptomatic episodes of gout, tophi, or radiographic changes of gout (55). But our analysis demonstrated that hyperuricemia was associated with lower eGFR, higher risk of graft loss, death and cardiovascular events. In addition, studies stated that using medication, like xanthine oxidase inhibition, was shown to reverse endothelial dysfunction (56), improve coronary and peripheral endothelial function (57), slow the GFR decline (58), defer the deterioration of renal dysfunction in CKD (59). Therefore, strengthening the management of asymptomatic hyperuricemia is needed. It is worth noting that low levels of serum UA appear also to contribute to poor clinical outcomes (21). The treatment of hyperuricemia should perhaps give priority to maintaining an appropriate serum UA level, rather than to simply lowering serum UA. We believe that it will be the next developments in the management for KTRs. Our review has the following limitations: first, all included studies were retrospective cohort studies, which might bring 
estimation bias. Second, some studies have shown that dietary factors are associated with the hyperuricemic group, however these dietary factors have not been evaluated in most of the included studies. Third, since the study population was from different transplant centers and uric acid measurements were from different laboratories, there may be variability in uric acid values. Fourth, although the included studies have been adjusted for confounding factors, the specific adjustment factors are different which may lead to a biased estimation of the results.

\section{CONCLUSION}

Our analysis found that increased uric acid levels contributed to the long-term decline of eGFR, the risk of graft loss and death increase after transplantation. Hyperuricemia increased the risk of cardiovascular event with no significant difference in the level of triglyceride between the two groups. Future research is needed to verify whether lowering uric acid level could improve the kidney function and prognosis of RTRs with hyperuricemia.

ETHICAL APPROVAL. This study was a systematic review and meta-analysis and based on the published articles, so ethical approval was not required.

CONFLICT OF INTEREST. No conflict of interest to report.

FUNDING DISCLOSURE. This research did not receive any specific grant from funding agencies in the public, commercial, or not-for-profit sectors.

CONTRIBUTORS. HY, and LHL designed the experiments, and QC, AWH, XJY, GC, XPH, WW, HL and XDZ collected and analyzed the data. This article was written by HY and QC. All authors reviewed the article. Hui Yang and Qing Chen are coprincipal authors and have contributed equally.

ACKNOWLEDGMENTS. We thank all the original authors of the included studies for their wonderful work.

\section{REFERENCES}

1. Clive DM. Renal transplant-associated hyperuricemia and gout. J Am Soc Nephrol. 2000; 11(5): 974-979. doi:10.1681/ASN.V115974.

2. Mazali FC, Mazzali M. Uric acid and transplantation. Semin Nephrol. 2011;31(5): 466-471.

doi: 10.1016/j.semnephrol.2011.08.012.

3. Han M, Lee JP, Park S, et al. Early onset hyperuricemia is a prognostic marker for kidney graft failure: Propensity score matching analysis in a Korean multicenter cohort. PLoS One. 2017; 12(6): e0179779. doi: 10.1371/journal.pone.0179779.

4. Srivastava A, Kaze AD, McMullan CJ, et al. Uric Acid and the Risks of Kidney Failure and Death in Individuals With CKD. Am J Kidney Dis. 2018; 71(3): 362-370. doi: 10.1053/j.ajkd.2017.08.017.

5. Huang Y, Li YL, Huang H, Wang L, et al. Effects of hyperuricemia on renal function of renal transplant recipients: a systematic review and meta-analysis of cohort studies. PLoS One. 2012; 7(6): e39457. doi: 10.1371/journal.pone.0039457.

6. Biyik Z, Guney I. Relationship Between Uric Acid, Proteinuria, and Atherogenic Index of Plasma in Renal Transplant Patients. Transplant Proc. 2018; 50(10): 3376-3380. doi: 10.1016/j.transproceed.2018.05.021.

7. Kim DG, Choi HY, Kim HY, et al. Association between post-transplant serum uric acid levels and kidney transplantation outcomes. PLoS One. 2018; 13(12): e0209156. doi: 10.1371/journal.pone.0209156.

8. Ou S M, Tarng D C. The effect of uric acid levels on survival in kidney transplant recipients. Transpl Int. 2017; 30 (Suppl. 2): 234. doi: 10.1111/tri.13050.

9. Eyupoglu S, Eyupoglu D, Kendi-Celebi Z, et al. Risk Factors of Hyperuricemia After Renal Transplantation and Its Long-term Effects on Graft Functions. Transplant Proc. 2017; 49(3): 505-508.

doi: 10.1016/j.transproceed.2017.01.006.

10. Kalil RS, Carpenter MA, Ivanova A, et al. Impact of Hyperuricemia on Long-term Outcomes of Kidney Transplantation: Analysis of the FAVORIT Study. Am J Kidney Dis. 2017; 70(6): 762-769. doi: 10.1053/j.ajkd.2017.06.013.

11. Han M, Lee JP, Park S, et al. Early onset hyperuricemia is a prognostic marker for kidney graft failure: Propensity score matching analysis in a Korean multicenter cohort. PLoS One. 2017; 12(5): e0176786. doi: 10.1371/journal.pone.0176786.

12. Han M, Yang S, Jung I, et al. Early onset hyperuricemia is a prognostic marker for kidney allograft dysfunction. Am J Transplant, 
2016; $16 \quad$ (suppl $\quad 3): \quad 483 . \quad$ doi: 10.1111/ajt.13898

13. Oh IH, Kim JE, Lee CH, et al. A J-Shaped Association Between Serum Uric Acid Level and Allograft Outcomes After Living Donor Kidney Transplantation. Artif Organs. 2016; 40(2): 136-143. doi: 10.1111/aor.12519.

14. Kim ED, Famure O, Li Y, et al. Uric acid and the risk of graft failure in kidney transplant recipients: a re-assessment. Am J Transplant. 2015; 15(2): 482-488. doi: 10.1111/ajt.13000.

15. Zhang K, Gao B, Wang Y, et al. Serum Uric Acid and Renal Transplantation Outcomes: At Least 3-Year Post-transplant Retrospective Multivariate Analysis. PLoS One. 2015; 10(7): e0133834.

doi: 10.1371/journal.pone.0133834.

16. Muela HC, De Lima JJ, Gowdak LH, et al. Prognostic Value of Serum Uric Acid in Patients on the Waiting List before and after Renal Transplantation. Int J Nephrol. 2015; 2015: 375606. doi: 10.1155/2015/375606.

17. Erkmen Uyar M, Sezer S, Bal Z, et al. Posttransplant Hyperuricemia as a Cardiovascular Risk Factor. Transplant Proc. 2015; 47(4): 1146-1151.

doi: 10.1016/j.transproceed.2015.03.004.

18. Fidan C, Kantar A, Baskin E, et al. Effects of hyperuricemia on renal function in pediatric renal transplant recipients. Exp Clin Transplant. 2015; 13 Suppl 1: 247-250. doi: 10.6002/ect.mesot2014.P49.

19. Weng SC, Shu KH, Tarng DC, et al. Uric acid is highly associated with kidney allograft survival in a time-varying analysis. Transplant Proc. 2014; 46(2): 505-510. doi: 10.1016/j.transproceed.2013.09.038.

20. Weng SC, Shu KH, Wu MJ, et al. Hyperuricemia predicts kidney disease progression after acute allograft dysfunction. Transplant Proc. 2014; 46(2): 499-504. doi: 10.1016/j.transproceed.2013.09.040.

21. Dahle DO, Jenssen T, Holdaas H, et al. Uric acid has a J-shaped association with cardiovascular and all-cause mortality in kidney transplant recipients. Clin Transplant. 2014; 28(1): 134-140. doi: 10.1111/ctr.12290.

22. Choi JY, Kwon OJ. The association between serum uric acid levels at 3 months after renal transplantation and the graft outcome in living donor renal transplantation. Transplant Proc. 2013; 45(4): 1548-1552. doi: 10.1016/j.transproceed.2012.10.062.

23. Kim KM, Kim SS, Yun S, et al. Uric acid contributes to glomerular filtration rate deterioration in renal transplantation. Nephron Clin Pract. 2011; 118(2): c136-c142. doi: $10.1159 / 000320616$.

24. Caliskan Y, Gorgulu N, Yelken B, et al. Serum uric acid level is associated with cardiac hypertrophy in renal transplant recipients. Clin Transplant. 2011; 25(3): 368-374. doi: 10.1111/j.1399-0012.2010.01244.x.

25. Haririan A, Metireddy M, Cangro C, et al. Association of serum uric acid with graft survival after kidney transplantation: a timevarying analysis. Am $J$ Transplant. 2011; 11(9): 1943-1950. doi: 10.1111/j.16006143.2011.03613.x.

26. GA Wells, B Shea, D O'Connell, et al. The Newcastle-Ottawa Scale (NOS) for assessing the quality of nonrandomized studies in metaanalyses. Available:

http://www.ohri.ca/programs/clinical_epidem iology/oxford.htm.Accessed 20 September

2015.

27. Higgins JP, Altman DG, Gøtzsche PC, Jüni P, Moher D, Oxman AD, Savović J, Schulz KF, Weeks L, Sterne JA. The Cochrane Collaboration's tool for assessing risk of bias in randomised trials. BMJ. 2011;343:d5928. doi: 10.1136/bmj.d5928.

28. Irwig L, Macaskill P, Berry G, et al. Bias in meta-analysis detected by a simple, graphical test. Graphical test is itself biased. BMJ. 1998; 316(7129): 470-471. PMID: 9492687.

29. Zou GM, Sui WG, Yan Q, et al. Influence of hyperuricemia on long-term renal allograft function after renal transplantation Is it a factor of chronic renal allograft dysfunction? Journal of Clinical Rehabilitative Tissue Engineering Research. 2010; 14(5): 923-927. doi: 10.3969/j.issn.1673-8225.2010.05.040.

30. Chung BH, Kang SH, Hwang HS, et al. Clinical significance of early-onset hyperuricemia in renal transplant recipients. Nephron Clin Pract. 2011; 117(3): c276-c283. doi: 10.1159/000320743.

31. Haririan A, Nogueira JM, Zandi-Nejad K, et al. The independent association between serum uric acid and graft outcomes after kidney transplantation. Transplantation. 2010; 89(5): $\quad 573-579 . \quad$ doi: 10.1097/TP.0b013e3181c73c18.

32. Kim KM, Kim SS, Han DJ, et al. Hyperuricemia in kidney transplant recipients with intact graft function. Transplant Proc. 2010; 42(9): 3562-3567. doi: 10.1016/j.transproceed.2010.07.104.

33. Min SI, Yun IJ, Kang JM, et al. Moderate-to- 
severe early-onset hyperuricaemia: a prognostic marker of long-term kidney transplant outcome. Nephrol Dial Transplant. 2009; 24(8): 2584-2590. doi: 10.1093/ndt/gfp192.

34. Bandukwala F, Huang M, Zaltzman JS, et al. Association of uric acid with inflammation, progressive renal allograft dysfunction and post-transplant cardiovascular risk. $\mathrm{Am} J$ Cardiol. 2009; 103(6): 867-871. doi: 10.1016/j.amjcard.2008.11.042.

35. Karbowska A, Boratynska M, Kusztal M, et al. Hyperuricemia is a mediator of endothelial dysfunction and inflammation in renal allograft recipients. Transplant Proc. 2009; 41(8): 3052-3055. doi: 10.1016/j.transproceed.2009.07.080.

36. Akalin E, Ganeshan SV, Winston J, et al. Hyperuricemia is associated with the development of the composite outcomes of new cardiovascular events and chronic allograft nephropathy. Transplantation. 2008; 86(5): 652-658. doi: 10.1097/TP.0b013e3181814f5b.

37. Sullivan PM, William A, Tichy EM. Hyperuricemia and gout in solid-organ transplant: update in pharmacological management. Prog Transplant. 2015; 25(3): 263-270. doi: 10.7182/pit2015322.

38. Kato M, Hisatome I, Tomikura Y, et al. Status of endothelial dependent vasodilation in patients with hyperuricemia. Am J Cardiol. 2005; 96(11): 1576-1578. doi: 10.1016/j.amjcard.2005.07.068.

39. Ruggiero C, Cherubini A, Ble A, et al. Uric acid and inflammatory markers. Eur Heart $J$. 2006; 27(10): 1174-1181. doi: 10.1093/eurheartj/ehi879.

40. Kang DH, Nakagawa T, Feng L, et al. A role for uric acid in the progression of renal disease. J Am Soc Nephrol. 2002; 13(12): 2888-2897. doi: 10.1097/01.asn.0000034910.58454.fd.

41. Kanellis J, Kang DH. Uric acid as a mediator of endothelial dysfunction, inflammation, and vascular disease. Semin Nephrol. 2005; 25(1): 39-42.

doi: 10.1016/j.semnephrol.2004.09.007.

42. Gerhardt U, Grosse Hüttmann M, Hohage H. Influence of hyperglycemia and hyperuricemia on long-term transplant survival in kidney transplant recipients. Clin Transplant. 1999; 13(5): 375-379. doi: 10.1034/j.1399-0012.1999.130502.x.

43. Nakagawa T, Mazzali M, Kang DH, et al. Hyperuricemia causes glomerular hypertrophy in the rat. Am J Nephrol. 2003; 23(1): 2-7. doi: 10.1159/000066303.

44. Mazzali M, Kanellis J, Han L, et al. Hyperuricemia induces a primary renal arteriolopathy in rats by a blood pressureindependent mechanism. Am J Physiol Renal Physiol. 2002; 282(6): F991-F997. doi: 10.1152/ajprenal.00283.2001.

45. Khosla UM, Zharikov S, Finch JL, et al. Hyperuricemia induces endothelial dysfunction. Kidney Int. 2005; 67(5): 17391742. doi: 10.1111/j.1523-1755.2005.00273.x.

46. Choi HY, Kim SH, Choi AR, et al. Hyperuricemia and risk of increased arterial stiffness in healthy women based on health screening in Korean population. PLoS One. 2017; 12(6): e0180406. doi: 10.1371/journal.pone.0180406.

47. Canepa M, Viazzi F, Strait JB, et al. Longitudinal Association Between Serum Uric Acid and Arterial Stiffness: Results From the Baltimore Longitudinal Study of Aging. Hypertension. 2017; 69(2): 228-235. doi: 10.1161/HYPERTENSIONAHA.116.08114.

48. Jevnikar AM, Mannon RB. Late kidney allograft loss: what we know about it, and what we can do about it. Clin J Am Soc Nephrol. 2008; 3 Suppl 2(Suppl 2): S56-S67. doi: 10.2215/CJN.03040707.

49. Moreso $F$, Ibernon $M$, Gomà $M$, et al. Subclinical rejection associated with chronic allograft nephropathy in protocol biopsies as a risk factor for late graft loss. Am J Transplant. 2006; 6(4): 747-752. doi: 10.1111/j.16006143.2005.01230.x.

50. Fang J, Alderman MH. Serum uric acid level and cardiovascular mortality. The NHANES 1 epidemiologic follow-up study. JAMA. 2000; 283(18): 2404-2410. doi: 10.1001/jama.283.18.2404.

51. Santos RD, Nasir K, Orakzai R, et al. Relation of uric acid levels to presence of coronary artery calcium detected by electron beam tomography in men free of symptomatic myocardial ischemia with versus without the metabolic syndrome. Am J Cardiol. 2007; 99(1): 42-45.

doi: 10.1016/j.amjcard.2006.07.057.

52. Viazzi F, Parodi D, Leoncini G, et al. Serum uric acid and target organ damage in primary hypertension. Hypertension. 2005; 45(5): 991 996.

doi: 10.1161/01.HYP.0000161184.10873.ea.

53. Corry DB, Eslami P, Yamamoto K, et al. Uric acid stimulates vascular smooth muscle cell 
proliferation and oxidative stress via the vascular renin-angiotensin system. $J$ Hypertens. 2008; 26(2): 269-275. doi: 10.1097/HJH.0b013e3282f240bf.

54. Feig DI, Kang DH, Johnson RJ. Uric acid and cardiovascular risk. $N$ Engl J Med. 2008; 359(17): 1811-1821. doi: 10.1056/NEJMra0800885.

55. Kidney Disease: Improving Global Outcomes (KDIGO) Transplant Work Group. KDIGO clinical practice guideline for the care of kidney transplant recipients. Am J Transplant. 2009; 9 Suppl 3: S1-S155. doi: 10.1111/j.1600-6143.2009.02834.x.

56. Guthikonda S, Sinkey C, Barenz T, et al. Xanthine oxidase inhibition reverses endothelial dysfunction in heavy smokers. Circulation. 2003; 107(3): 416-421. doi: 10.1161/01.cir.0000046448.26751.58.
57. Baldus S, Koster R, Chumley $\mathrm{P}$, et al. Oxypurinol improves coronary and peripheral endothelial function in patients with coronary artery disease. Free Radic Biol Med. 2005; 39(9): 1184-90. doi: 10.1016/j.freeradbiomed.2005.06.004.

58. Sircar D, Chatterjee S, WaikhomR, et al. Efficacy of Febuxostat for Slowing the GFR Decline in Patients With CKD and Asymptomatic Hyperuricemia: A 6-Month, Double-Blind, Randomized, PlaceboControlled Trial. Am J Kidney Dis. 2015; 66(6): 945-950. doi: 10.1053/j.ajkd.2015.05.017.

59. Siu YP, Leung KT, Tong MK, et al. Use of allopurinol in slowing the progression of renal disease through its ability to lower serum uric acid level. Am J Kidney Dis. 2006; 47(1):519. doi: 10.1053/j.ajkd.2005.10.006 


\section{SUPPLEMENTARY DOCUMENTS}

\section{Table 1s}

Pubmed: Search $\quad((((()((()((($ urate[Title/Abstract]) $\quad$ OR uric acid[Title/Abstract]) OR urea acid[Title/Abstract]) OR acid urate[Title/Abstract]) OR gout[Title/Abstract])) OR "Uric Acid"[Mesh]) OR "Hyperuricemia"[Mesh]) OR hyperuric[Title/Abstract]) OR hyperuricemia[Title/Abstract]) OR hyperuricaemia[Title/Abstract])) AND ((((((("Transplantation"[Mesh]) OR "Transplants"[Mesh]) OR transplants[Title/Abstract]) OR transplant[Title/Abstract]) OR transplantation[Title/Abstract]) OR transplantations[Title/Abstract]) OR graft[Title/Abstract]))) AND ("2011/6"[Date - Publication] : "3000"[Date - Publication])

\section{Cochrane library:}

\#1 MeSH descriptor: [Hyperuricemia] explode all trees

\#2 (hyperuricemia):ti,ab,kw (Word variations have been searched)

\#3 (hyperuricaemia):ti,ab,kw (Word variations have been searched)

\#4 (urea acid):ti,ab,kw (Word variations have been searched)

\#5 (Uric acid):ti,ab,kw (Word variations have been searched)

\#6 (acid urate):ti,ab,kw (Word variations have been searched)

\#7 (urate):ti,ab,kw (Word variations have been searched)

\#8 (gout):ti,ab,kw (Word variations have been searched)

\#9 \#1 or \#2 or \#3 or \#4 or \#5 or \#6 or \#7 or \#8 6582

\#10 MeSH descriptor: [Uric Acid] explode all trees

\#11 (hyperuric):ti,ab,kw (Word variations have been searched)

\#12 \#9 or \#10 or \#11 6582

\#13 MeSH descriptor: [Transplantation] explode all trees

\#14 MeSH descriptor: [Transplants] explode all trees

\#15 (transplant):ti,ab,kw (Word variations have been searched)

\#16 (transplants):ti,ab,kw (Word variations have been searched)

\#17 (transplantation):ti,ab,kw (Word variations have been searched)

\#18 (transplantations):ti,ab,kw (Word variations have been searched)

\#19 (graft):ti,ab,kw (Word variations have been searched)

\#20 \#13 or \#14 or \#15 or \#16 or \#17 or \#18 or \#19

\#21 \#12 and \#20

\#22 \#12 and \#20 with Cochrane Library publication date to Mar 2020

\section{EMBASE:}

\#21. \#11AND\#19AND[2011-2020]/py

\#20. \#11AND\#19

\#19. \#12OR\#13OR\#14OR\#15OR\#16OR\#17

\#18. 'transplants'/exp OR transplants

\#17. graft:ab,ti 20 Mar 2020

\#16. transplants:ab,ti 20 Mar 2020

\#15. transplant:ab,ti 20 Mar 2020

\#14. transplantations:ab,ti 20 Mar 2020

\#13.'transplantation':ab,ti 20 Mar 2020 
\#12.'transplantation'/exp 20 Mar 2020

\#11. \#1 OR \#2 OR \#3 OR \#4 OR \#5 OR \#6 OR \#7 OR \#8 OR \#9 OR \#10 20 Mar 2020

\#10.'gout':ab,ti 20 Mar 2020

\#9. hyperuric:ab,ti 20 Mar 2020

\#8. hyperuricaemia:ab,ti

\#7.'hyperuricemia':ab,ti

20 Mar 2020

\#6.'hyperuricemia'/exp

20 Mar 2020

\#5.'acid urate':ab,ti

20 Mar 2020

\#4.'urea acid':ab,ti

20 Mar 2020

\#3.'uric acid':ab,ti

20 Mar 2020

\#2. urate:ab,ti

20 Mar 2020

\#1.'uric acid'/exp

20 Mar 2020

Table 2s. Quality of observational studies (indicators from New-Castle-Ottawa scale)

\begin{tabular}{|c|c|c|c|c|c|c|c|c|c|c|}
\hline Reference & $1 \mathbf{a}$ & $2 \mathbf{b}$ & $3 c$ & $4 d$ & $5 \mathrm{Ae}$ & $5 \mathrm{Bf}$ & $6 \mathrm{~g}$ & $7 \mathrm{~h}$ & $8 \mathbf{i}$ & $\begin{array}{l}\text { Total quality } \\
\text { scores }\end{array}$ \\
\hline 6 & Yes & Yes & Yes & Yes & No & No & Yes & Yes & Yes & 7 \\
\hline 7 & Yes & Yes & Yes & Yes & No & No & Yes & Yes & Yes & 7 \\
\hline 8 & Yes & Yes & Yes & Yes & No & No & Yes & Yes & Yes & 7 \\
\hline 9 & Yes & Yes & Yes & Yes & Yes & No & Yes & Yes & Yes & 8 \\
\hline 10 & Yes & Yes & Yes & Yes & Yes & No & Yes & Yes & Yes & 8 \\
\hline 11 & Yes & Yes & Yes & Yes & Yes & Yes & Yes & Yes & Yes & 9 \\
\hline 12 & Yes & Yes & Yes & Yes & No & No & Yes & Yes & Yes & 7 \\
\hline 13 & Yes & Yes & Yes & Yes & Yes & Yes & Yes & Yes & Yes & 9 \\
\hline 14 & Yes & Yes & Yes & Yes & Yes & Yes & Yes & Yes & Yes & 9 \\
\hline 15 & Yes & Yes & Yes & Yes & Yes & Yes & Yes & Yes & Yes & 9 \\
\hline 16 & Yes & Yes & Yes & Yes & Yes & Yes & Yes & Yes & Yes & 9 \\
\hline 17 & Yes & Yes & Yes & Yes & Yes & No & Yes & Yes & Yes & 8 \\
\hline 18 & Yes & Yes & Yes & Yes & Yes & No & Yes & Yes & Yes & 8 \\
\hline 19 & Yes & Yes & Yes & Yes & No & No & Yes & Yes & Yes & 7 \\
\hline 20 & Yes & Yes & Yes & Yes & Yes & Yes & Yes & Yes & Yes & 9 \\
\hline 21 & Yes & Yes & Yes & Yes & Yes & Yes & Yes & Yes & Yes & 9 \\
\hline 22 & Yes & Yes & Yes & Yes & Yes & No & Yes & Yes & Yes & 8 \\
\hline 23 & Yes & Yes & Yes & Yes & Yes & No & Yes & Yes & Yes & 8 \\
\hline 24 & Yes & Yes & Yes & Yes & Yes & Yes & Yes & Yes & Yes & 9 \\
\hline 25 & Yes & Yes & Yes & Yes & Yes & Yes & Yes & Yes & Yes & 9 \\
\hline 29 & Yes & Yes & Yes & Yes & Yes & No & Yes & Yes & Yes & 8 \\
\hline 30 & Yes & Yes & Yes & Yes & Yes & Yes & Yes & Yes & Yes & 9 \\
\hline 31 & Yes & Yes & Yes & Yes & Yes & Yes & Yes & Yes & Yes & 9 \\
\hline 32 & Yes & Yes & Yes & Yes & Yes & No & Yes & Yes & Yes & 8 \\
\hline 33 & Yes & Yes & Yes & Yes & Yes & No & Yes & Yes & Yes & 8 \\
\hline 34 & Yes & Yes & Yes & Yes & Yes & No & Yes & Yes & Yes & 8 \\
\hline 35 & Yes & Yes & Yes & Yes & Yes & No & Yes & Yes & Yes & 8 \\
\hline 36 & Yes & Yes & Yes & Yes & Yes & Yes & Yes & Yes & Yes & 9 \\
\hline
\end{tabular}

a: Indicates exposed cohort truly representative; b: Non-exposed cohort drawn from the same community; c: Ascertainment of exposure from the same community; d: Outcome of interest not present at start of study; e: Cohorts comparable on basis of site and etiology of infection; f: Cohorts comparable on others factors; g: Assessment of outcome of record linkage or independent blind assessment; h: Follow-up long enough for outcomes to occur; I: Complete accounting for cohort. 